\title{
On the Second-Order Statistics of Correlated Cascaded Rayleigh Fading Channels
}

\author{
Yawgeng A. Chau and Karl Y.-T. Huang \\ Department of Communications Engineering, Yuan Ze University, 135 Yuan-Tung Road, Chung-Li 32003, Taiwan \\ Correspondence should be addressed to Yawgeng A. Chau, eeyaw@saturn.yzu.edu.tw
}

Received 10 November 2011; Accepted 7 June 2012

Academic Editor: Matteo Pastorino

Copyright ( $) 2012$ Y. A. Chau and K. Y.-T. Huang. This is an open access article distributed under the Creative Commons Attribution License, which permits unrestricted use, distribution, and reproduction in any medium, provided the original work is properly cited.

The second-order statistics of two correlated cascaded (double) Rayleigh fading channels are analyzed, where different relevant second-order cross-correlation functions of in-phase and quadrature components of the cascaded Rayleigh channels are derived. The level crossing rate (LCR) and average fade duration (AFD) of the cascaded channels are evaluated, and a single-integral form of the LCR is derived. Numerical results of the LCR and AFD are presented, and the effect of the correlation is illustrated.

\section{Introduction}

Recently, the cascaded fading channels have attracted a lot of research interests [1-12]. The cascaded fading channels can be used to model different wireless communication scenarios, such as the keyhole fading channel [4], the mobile-to-mobile transmission channel [5], dual-hop fading channels [8], and radio frequency identification (RFID) pinhole channels [9-12].

In the published works of [1-9], the cascaded processes are assumed to be independent. For cascaded keyhole or pinhole fading channels, since the faded signals terminate and originate at the same keyhole or pinhole location, the correlation between cascaded fading processes may have some impacts on the performance of wireless transmissions. In [10-12], the bit error rate (BER) of RFID with multiple tags has been examined for correlated cascaded (forwardbackscatter) channels. In fact, the completely dependent or correlated cascaded Rayleigh fading channels have been studied in earlier works [13], where the corresponding BERs of different modulations have been evaluated. The scenarios of keyhole multiple-input multiple-output (MIMO) channels and RFID pinhole channels with a correlation coefficient are illustrated in Figure 1.

In this paper, in contrast to the evaluation of first-order performance for correlated cascaded Rayleigh channels, such as the bit error rate given in [10-12], second-order statistics of correlated cascaded Rayleigh processes are analyzed and evaluated. The second-order statistics such as the LCR and AFD are useful for the design of practical wireless communication systems [14-19]. The LCR of a faded signal is defined as the average rate at which the signal envelope crosses a given level, and the AFD is the average duration that the signal envelope becomes lower than the given threshold $[14,15]$. As addressed in [20], the second-order statistics, LCR and AFD, are also important for the design of real RFID systems. In [8], the LCR and AFD were analyzed for independent cascaded Rayleigh fading channels. In [16], the LCR and AFD were applied to the burst-error analysis for independent cascaded Rayleigh fading channels. In [17], the LCR and AFD of cooperative selection diversity were studied, where independent cascaded Rayleigh fading channels were assumed. In [18, 19], the LCR and AFD were evaluated for independent cascaded Nakagami- $m$ fading channels.

In the context, two completely correlated Rayleigh processes [13] in cascaded are modelled, corresponding second-order correlation functions are derived, and the LCR and AFD of the correlated cascaded Rayleigh processes are evaluated. The result of the paper can be applied to the analysis of second-order statistics of correlated cascaded Rayleigh fading channels, such as a path of keyhole or pinhole fading channels shown in Figure 1. 


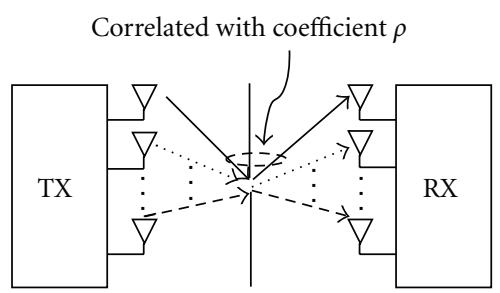

(a) Keyhole MIMO channels

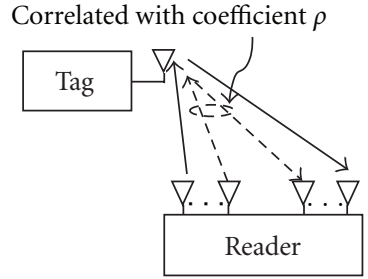

(b) RFID pinhole channels

FIGURE 1: The scenarios of keyhole MIMO channels and RFID pinhole channels.

In Section 2, the correlated cascaded Rayleigh random processes and their in-phase and quadrature processes are modelled, where various second-order correlation functions are derived. In Section 3, the LCR and AFD of the correlated cascaded processes are evaluated. In Section 4, numerical results of the LCR and AFD are given and the effect of the correlation is illustrated. Conclusions are drawn in Section 5.

\section{Second-Order Correlation Functions of Correlated Cascaded Rayleigh Processes}

2.1. Modeling. Let $\alpha_{i}(t)(i=1,2)$ be a Rayleigh process with the probability density function (pdf) given by the following:

$$
f_{i}\left(\alpha_{i}\right)=\frac{\alpha_{i}}{\sigma_{i}^{2}} e^{-\alpha_{i}^{2} /\left(2 \sigma_{i}^{2}\right)}, \quad \alpha_{i} \geq 0,
$$

where $2 \sigma_{i}^{2}=E\left[\alpha_{i}^{2}\right]$. Then, two correlated Rayleigh processes in cascade can be modelled by the following:

$$
R(t, \tau)=\alpha_{1}(t) \alpha_{2}(t+\tau), \quad t, \tau \geq 0,
$$

where $\tau$ is the time or phase offset between $\alpha_{1}$ and $\alpha_{2}$. The joint pdf of $\alpha_{1}$ and $\alpha_{2}$ is [21]

$$
\begin{aligned}
f_{12}\left(\alpha_{1}, \alpha_{2}\right)= & \frac{\alpha_{1} \alpha_{2}}{\sigma_{1}^{2} \sigma_{2}^{2}\left(1-\rho^{2}\right)} e^{-\left(\sigma_{2}^{2} \alpha_{1}^{2}+\sigma_{1}^{2} \alpha_{2}^{2}\right) /\left(2 \sigma_{1}^{2} \sigma_{2}^{2}\left(1-\rho^{2}\right)\right)} \\
& \times I_{0}\left(\frac{\alpha_{1} \alpha_{2}|\rho|}{\sigma_{1} \sigma_{2}\left(1-\rho^{2}\right)}\right), \quad \alpha_{1}, \alpha_{2} \geq 0,
\end{aligned}
$$

where $I_{0}(\cdot)$ is the zeroth order modified Bessel function of the first kind, and the correlation coefficient is in the range $-1<\rho<1$. Notice that when $\rho=1, \alpha_{1}$ and $\alpha_{2}$ have a linear relation, and $R$ reduces to the well-known chi-square distribution. With $f_{12}\left(\alpha_{1}, \alpha_{2}\right)$ given by (3), it is straightforward to show that the pdf of $R$ is

$$
\begin{aligned}
f_{R}(r) & =\int_{0}^{\infty} \frac{1}{\alpha_{2}} f_{12}\left(\frac{r}{\alpha_{2}}, \alpha_{2}\right) d \alpha_{2} \\
& =\frac{r}{\sigma_{1}^{2} \sigma_{2}^{2}\left(1-\rho^{2}\right)} I_{0}\left(\frac{r|\rho|}{\sigma_{1} \sigma_{2}\left(1-\rho^{2}\right)}\right) K_{0}\left(\frac{r}{\sigma_{1} \sigma_{2}\left(1-\rho^{2}\right)}\right),
\end{aligned}
$$

where $K_{0}(\cdot)$ is the zeroth order modified Bessel function of the second kind, and $[22,(3.478 .4)]$ is used to evaluate the integral. In Figure 2, the pdf with different correlation coefficients is shown. From Figure 2, when $\rho$ increases, the cascaded Rayleigh processes has a larger probability of yielding smaller values.

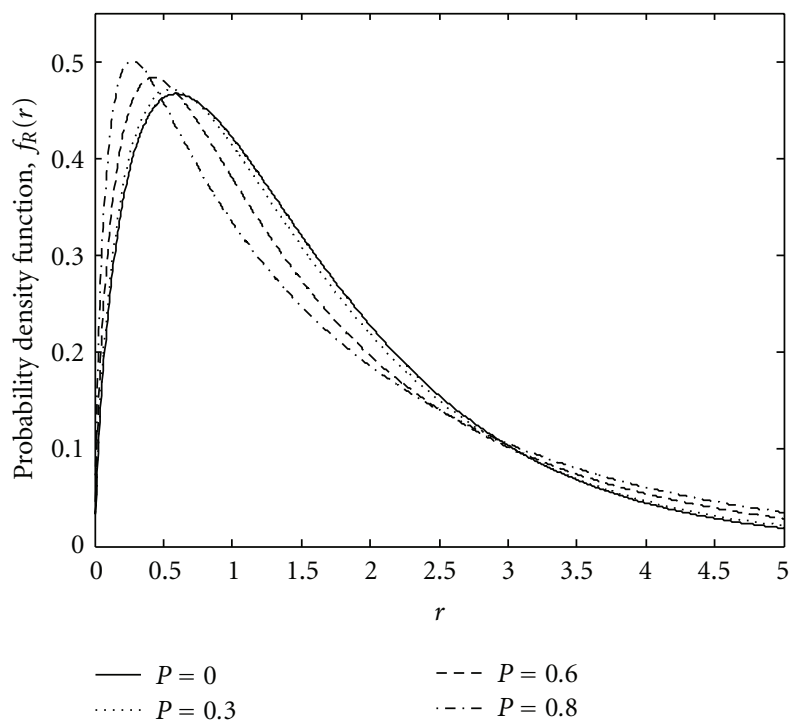

FIGURE 2: Probability density function of correlated cascade Rayleigh processes.

2.2. Second-Order Correlation Functions of In-Phase and Quadrature Processes. For $i=1,2$, let $X_{i}$ and $Y_{i}$ be the underlying in-phase and quadrature components of $\alpha_{i}(t)$, and

$$
\alpha_{i}^{2}(t)=X_{i}^{2}(t)+Y_{i}^{2}(t)
$$

The time derivatives of $X_{i}, Y_{i}$, and $\alpha_{i}(t)$ give the second-order information of cascaded Rayleigh processes.

Le $f_{m}$ be the maximal Doppler frequency of the cascaded fading channels and $\theta_{k}$ be the uniformly distributed phase on $[0,2 \pi]$. Based on the modeling used in $[13,14]$, for $i=1,2$, we may write $\left(X_{i}, Y_{i}\right)$ in the following forms [23]

$$
\begin{aligned}
& X_{i}(t)=\lim _{n \rightarrow \infty} \sqrt{2 \sigma_{i}^{2}} \sum_{k=1}^{n} a_{k} \cos \left(\omega_{k} t-\omega_{c, i} T_{k}\right), \\
& Y_{i}(t)=\lim _{n \rightarrow \infty} \sqrt{2 \sigma_{i}^{2}} \sum_{k=1}^{n} a_{k} \sin \left(\omega_{k} t-\omega_{c, i} T_{k}\right),
\end{aligned}
$$

where $\omega_{k}=2 \pi f_{m} \cos \theta_{k}, a_{k}$ is the corresponding fractional power for the $k$ th component, $\omega_{c, i}$ is the frequency, and $T_{k}$ is the time-delay [15]. In practice, $T_{k}$ can be modelled by 
an exponential distribution with mean $\bar{T}$ [15]. The time derivatives of $\left(X_{i}, Y_{i}\right)$ are

$$
\begin{gathered}
\dot{X}_{i}(t)=-\lim _{n \rightarrow \infty} \sqrt{2 \sigma_{i}^{2}} \sum_{k=1}^{n} a_{k} \omega_{k} \cos \theta_{k} \sin \left(\omega_{k} t-\omega_{c, i} T_{k}\right), \\
\dot{Y}_{i}(t)=\lim _{n \rightarrow \infty} \sqrt{2 \sigma_{i}^{2}} \sum_{i=1}^{n} a_{k} \omega_{k} \cos \theta_{k} \cos \left(\omega_{k} t-\omega_{c, i} T_{k}\right) .
\end{gathered}
$$

Let $\langle\cdot\rangle$ denote the average operator. With (7), we can directly obtain that the second-order autocorrelation functions are

$$
\left\langle X_{i}(t) \dot{X}_{i}(t+\tau)\right\rangle=\left\langle Y_{i}(t) \dot{Y}_{i}(t+\tau)\right\rangle=0, \quad i=1,2 .
$$

Similarly, the second-order intercross-correlation functions of the in-phase and quadrature processes can be obtained as

$$
\begin{aligned}
\left\langle X_{i}(t) \dot{Y}_{i}(t+\tau)\right\rangle & =\left\langle\dot{X}_{i}(t) Y_{i}(t+\tau)\right\rangle \\
& =\left\langle\dot{X}_{i}(t) \dot{Y}_{i}(t+\tau)\right\rangle=0, \quad i=1,2 .
\end{aligned}
$$

Let $\Delta \omega=\omega_{c, 2}-\omega_{c, 1}$ and $\omega_{m}=2 \pi f_{m}$. By using (7) and some manipulations, the second-order cross-correlation function $\left\langle\dot{X}_{1}(t) \dot{X}_{2}(t+\tau)\right\rangle$ and $\left\langle\dot{Y}_{1}(t) \dot{Y}_{2}(t+\tau)\right\rangle$ can be derived as

$$
\begin{aligned}
\left\langle\dot{X}_{1}(t) \dot{X}_{2}(t+\tau)\right\rangle= & \left\langle\dot{Y}_{1}(t) \dot{Y}_{2}(t+\tau)\right\rangle \\
= & \sigma_{1} \sigma_{2} \int_{0}^{2 \pi} \int_{0}^{\infty} \frac{e^{-T_{k} / T}}{2 \pi \bar{T}} \\
& \times \cos \left(\omega_{m} \tau \cos \theta_{k}-\Delta \omega T_{k}\right) d \theta_{k} d T_{k} \\
& +\sigma_{1} \sigma_{2} \omega_{m}^{2} \int_{0}^{2 \pi} \int_{0}^{\infty} \frac{e^{-T_{k} / \bar{T}}}{4 \pi \bar{T}} \\
& \times \cos 2 \theta_{k} \cos \left(\Delta \omega T_{k}\right) \\
& \times \cos \left(\omega_{m} \tau \cos \theta_{k}\right) d \theta_{k} d T_{k} \\
& +\sigma_{1} \sigma_{2} \omega_{m}^{2} \int_{0}^{2 \pi} \int_{0}^{\infty} \frac{e^{-T_{k} / \bar{T}}}{4 \pi \bar{T}} \\
& \times \cos 2 \theta_{k} \sin \left(\Delta \omega T_{k}\right) \\
& \times \sin \left(\omega_{m} \tau \cos \theta_{k}\right) d \theta_{k} d T_{k} \\
= & \frac{\sigma_{1} \sigma_{2} \omega_{m}^{2}}{2\left[1+(\Delta \omega \bar{T})^{2}\right]} \\
& \times\left[J_{0}\left(\omega_{m} \tau\right)-J_{2}\left(\omega_{m} \tau\right)\right],
\end{aligned}
$$

where $J_{i}(\cdot)(i=0,1,2, \ldots)$ denotes the $i$ th-order Bessel function of the first kind, and [22, (3.715), (8.339), (3.895), and (8.473.1)] are applied to evaluate the integral.

When the two cascaded channels are using the same carrier frequency, we have $\Delta \omega=0$. In Figure 3, this secondorder cross-correlation of in-phase and quadrature processes with $\Delta \omega=0$ is plotted for $\sigma_{1}^{2}=\sigma_{2}^{2}=1$. From Figure 2, the vibration range of the cross-correlation may increase when $f_{m}$ becomes higher.

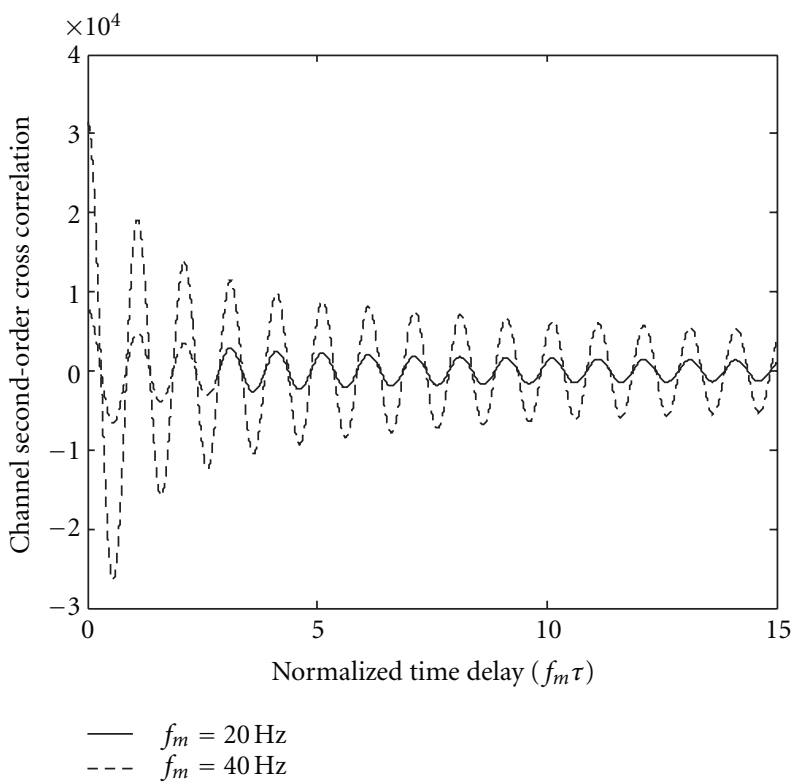

FIGURE 3: Cross-correlation function of in-phase and quadrature processes of the cascaded fading channels.

Other non-zero second-order correlation functions can be derived in a similar way. Following manipulations similar to those used for deriving (10), we can obtain the related second-order correlation functions as follows:

$$
\begin{aligned}
\left\langle\dot{X}_{1}(t) \dot{Y}_{2}(t+\tau)\right\rangle= & -\left\langle\dot{Y}_{1}(t) \dot{X}_{2}(t+\tau)\right\rangle \\
= & -\frac{\sigma_{1} \sigma_{2} \omega_{m}^{2} \Delta \omega \bar{T}}{2\left[1+(\Delta \omega \bar{T})^{2}\right]} \\
& \times\left[J_{0}\left(\omega_{m} \tau\right)-J_{2}\left(\omega_{m} \tau\right)\right], \\
\left\langle\dot{X}_{1}(t) X_{2}(t+\tau)\right\rangle= & -\left\langle X_{1}(t) \dot{X}_{2}(t+\tau)\right\rangle \\
= & -\left\langle\dot{Y}_{1}(t) Y_{2}(t+\tau)\right\rangle \\
= & -\left\langle Y_{1}(t) \dot{Y}_{2}(t+\tau)\right\rangle \\
= & -\frac{\sigma_{1} \sigma_{2} \omega_{m} J_{1}\left(\omega_{m} \tau\right)}{1+(\Delta \omega \bar{T})^{2}}, \\
\left\langle X_{1}(t) \dot{Y}_{2}(t+\tau)\right\rangle= & \left\langle\dot{Y}_{1}(t) X_{2}(t+\tau)\right\rangle \\
= & -\left\langle\dot{X}_{1}(t) Y_{2}(t+\tau)\right\rangle \\
= & -\left\langle Y_{1}(t) \dot{X}_{2}(t+\tau)\right\rangle \\
= & \frac{\sigma_{1} \sigma_{2} \omega_{m} \Delta \omega \bar{T} J_{1}\left(\omega_{m} \tau\right)}{1+(\Delta \omega \bar{T})^{2}} .
\end{aligned}
$$

The above second-order correlation functions can be applied to the derivation of the cross-correlation between $\dot{\alpha}_{1}(t)$ and $\dot{\alpha}_{2}(t+\tau)$ that will be useful for the LCR and AFD derivation in Section 3. 
2.3. Cross-Correlation Function of $\dot{\alpha}_{1}(t)$ and $\dot{\alpha}_{2}(t)$. For $i=$ 1,2 the time derivative of $\alpha_{i}(t)$ has the form

$$
\dot{\alpha}_{i}(t)=\frac{X_{i}(t) \dot{X}_{i}(t)+Y_{i}(t) \dot{Y}_{i}(t)}{\sqrt{X_{i}^{2}(t)+Y_{i}^{2}(t)}}=\frac{X_{i}(t) \dot{X}_{i}(t)+Y_{i}(t) \dot{Y}_{i}(t)}{\alpha_{i}(t)} .
$$

The correlation function between $\dot{\alpha}_{1}(t)$ and $\dot{\alpha}_{2}(t+\tau)$ under fixed $\alpha_{1}(t)$ and $\alpha_{2}(t+\tau)$ can be written as follows:

$$
\begin{aligned}
& \left\langle\dot{\alpha}_{1}(t) \dot{\alpha}_{2}(t+\tau)\right\rangle \\
& =\frac{\left\langle X_{1} \dot{X}_{1} X_{2} \dot{X}_{2}\right\rangle+\left\langle Y_{1} \dot{Y}_{1} Y_{2} \dot{Y}_{2}\right\rangle+\left\langle X_{1} \dot{X}_{1} Y_{2} \dot{Y}_{2}\right\rangle+\left\langle X_{2} \dot{X}_{2} Y_{1} \dot{Y}_{1}\right\rangle}{\alpha_{1}(t) \alpha_{2}(t+\tau)},
\end{aligned}
$$

where the variables $t$ in $\left(X_{1}, Y_{1}\right)$ and $(t+\tau)$ in $\left(X_{2}, Y_{2}\right)$ are omitted for simplification. To derive $\left\langle\dot{\alpha}_{1}(t) \dot{\alpha}_{2}(t+\tau)\right\rangle$, we need to obtain the four expectations in the numerator of (13). Based on the model of central limit theorem considered in $[14,15],[24,(8.111)]$, all $X_{i}, \dot{X}_{i}, Y_{i}$, and $\dot{Y}_{i}$ have a zeromean Gaussian distribution. On the other hand, it is well known that if the random variables $\left(Z_{1}, Z_{2}, Z_{3}, Z_{4}\right)$ have a jointly zero-mean Gaussian distribution, $\left\langle Z_{1} Z_{2} Z_{3} Z_{4}\right\rangle$ can be evaluated by $[19,(8.61)]$ the following:

$$
\begin{aligned}
\left\langle Z_{1} Z_{2} Z_{3} Z_{4}\right\rangle= & \left\langle Z_{1} Z_{2}\right\rangle\left\langle Z_{3} Z_{4}\right\rangle \\
& +\left\langle Z_{1} Z_{3}\right\rangle\left\langle Z_{2} Z_{4}\right\rangle \\
& +\left\langle Z_{1} Z_{4}\right\rangle\left\langle Z_{2} Z_{3}\right\rangle .
\end{aligned}
$$

Thus, we can use the property given by (14) to analyze the numerator of (13). The four expectations in the numerator of (13) can be derived by using (14) and the expectations of the forms $\left\langle Z_{i} Z_{j}\right\rangle$ and $\left\langle Z_{i} \dot{Z}_{j}\right\rangle(i, j=1,2)$. For $Z=X$ or $Y$ in (14), we have

$$
\begin{gathered}
\left\langle X_{i}(t) Y_{i}(t+\tau)\right\rangle=0, \\
\left\langle X_{1}(t) X_{2}(t+\tau)\right\rangle=\left\langle Y_{1}(t) Y_{2}(t+\tau)\right\rangle=\frac{\sigma_{1} \sigma_{2} J_{0}\left(\omega_{m} \tau\right)}{1+(\Delta \omega \bar{T})^{2}}, \\
\left\langle X_{1}(t) Y_{2}(t+\tau)\right\rangle=-\left\langle X_{2}(t) Y_{1}(t+\tau)\right\rangle=\frac{-\Delta \omega \bar{T} \sigma_{1} \sigma_{2} J_{0}\left(\omega_{m} \tau\right)}{1+(\Delta \omega \bar{T})^{2}} .
\end{gathered}
$$

Using the result obtained in Section 2.2, and (13)-(15), we can simplify (13) into the form

$$
\begin{aligned}
\left\langle\dot{\alpha}_{1}(t) \dot{\alpha}_{2}(t+\tau)\right\rangle & =\frac{\sigma_{1}^{2} \sigma_{2}^{2} \omega_{m}^{2}}{\alpha_{1}(t) \alpha_{2}(t+\tau)\left[1+(\Delta \omega \bar{T})^{2}\right]} \\
& \times\left[J_{0}^{2}\left(\omega_{m} \tau\right)-2 J_{1}^{2}\left(\omega_{m} \tau\right)-J_{0}\left(\omega_{m} \tau\right) J_{2}\left(\omega_{m} \tau\right)\right] .
\end{aligned}
$$

To evaluate (16), we employ the following relation derived in $[15,(1.5 .20)]$ and $[23,(4.31 \mathrm{~b})]$ for $\rho$ and $\Delta \omega \bar{T}$

$$
\rho^{2}\left(\omega_{m} \tau\right)=\frac{J_{0}^{2}\left(\omega_{m} \tau\right)}{1+(\Delta \omega \bar{T})^{2}}
$$

From (17), for $\Delta \omega=0$, the correlation coefficient is a function of the maximum Doppler frequency and the phase offset between the cascaded processes. Substituting (17) with $\Delta \omega=0$, we can simplify (16) into the form

$$
\begin{aligned}
\left\langle\dot{\alpha}_{1}(t) \dot{\alpha}_{2}(t+\tau)\right\rangle & =\frac{-\sigma_{1}^{2} \sigma_{2}^{2}}{\alpha_{1}(t) \alpha_{2}(t+\tau)} \frac{d^{2}}{d \tau^{2}} J_{0}^{2}\left(\omega_{m} \tau\right) \\
& =\frac{-\sigma_{1}^{2} \sigma_{2}^{2} \omega_{m}^{2}\left(\rho^{2}\right)^{\prime \prime}}{\alpha_{1}(t) \alpha_{2}(t+\tau)} \\
& =\frac{-\sigma_{1}^{2} \sigma_{2}^{2} \omega_{m}^{2}\left(\rho^{2}\right)^{\prime \prime}}{R(t, \tau)},
\end{aligned}
$$

where we use the recursive relations of Bessel functions given by $[22,(8.473 .1),(8.473 .4)]$, and $\left(\rho^{2}\right)^{\prime \prime}$ is defined by

$$
\left(\rho^{2}\right)^{\prime \prime}=\left[\frac{d^{2} \rho^{2}(x)}{d x^{2}}\right]_{x=\omega_{m} \tau} .
$$

For the derivation of the LCR and AFD of the correlated cascaded Rayleigh channels, in the next section, without loss of generality, $\Delta \omega=0$ is considered.

\section{Derivation of LCR and AFD}

3.1. $L C R$. The average $\mathrm{LCR}$ of a random process $R$ at $R=r$ can be evaluated by $[14,15]$

$$
N_{R}(r)=\int_{0}^{\infty} \dot{r} f_{R, \dot{R}}(r, \dot{r}) d \dot{r}=f_{R}(r) \int_{0}^{\infty} \dot{r} f_{\dot{R} \mid R}(\dot{r} \mid r) d \dot{r}
$$

where $\dot{r}$ denotes the time derivative of $r$, and $f_{\dot{R} \mid R}(\dot{r} \mid r)$ is the pdf of the time derivative $\dot{R}$ conditioned on $R=r$.

To evaluate the LCR given by (20), we first characterize the probability distribution of $\dot{R}$ below, where

$$
\dot{R}(t, \tau)=\alpha_{2}(t+\tau) \dot{\alpha}_{1}(t)+\alpha_{1}(t) \dot{\alpha}_{2}(t+\tau) .
$$

Based on [15], $\dot{\alpha}_{i}$ is Gaussian distributed with zero mean and variance $\sigma_{\dot{\alpha}_{i}}^{2}=2 \pi^{2} f_{m}^{2} \sigma_{i}^{2}$. Consequently, conditioned on fixed $\left(\alpha_{1}, \alpha_{2}\right), \dot{R}$ is Gaussian distributed with zero mean and the variance

$$
\dot{\sigma}_{R}^{2}=\alpha_{2}^{2} \sigma_{\dot{\alpha}_{1}}^{2}+\alpha_{1}^{2} \sigma_{\dot{\alpha}_{2}}^{2}+2 \alpha_{1} \alpha_{2} \operatorname{cov}\left(\dot{\alpha}_{1}, \dot{\alpha}_{2}\right),
$$

where $\operatorname{cov}\left(\dot{\alpha}_{1}, \dot{\alpha}_{2}\right)$ denotes the covariance of $\dot{\alpha}_{1}(t)$ and $\dot{\alpha}_{2}(t+$ $\tau)$ under fixed $\left(\alpha_{1}, \alpha_{2}\right)$, and is given by (18) for $\Delta \omega=0$. Let $f_{\dot{R} \mid r, \alpha}\left(\dot{r} \mid \alpha_{1}, \alpha_{2}\right)$ denote the conditional pdf of $\dot{R}$ under fixed $\left(\alpha_{1}, \alpha_{2}\right)$. Thus,

$$
f_{\dot{R} \mid r, \alpha}\left(\dot{r} \mid \alpha_{1}, \alpha_{2}\right) \sim \operatorname{Gaussian}\left(0, \dot{\sigma}_{R}^{2}\right) .
$$

Let $f_{R \mid \alpha}\left(r \mid \alpha_{1}, \alpha_{2}\right)$ denote the conditional pdf of $R$ under fixed $\left(\alpha_{1}, \alpha_{2}\right)$. Then, for the correlated cascaded processes, the LCR given by (20) can be rewritten in the form

$$
\begin{aligned}
N_{R}(r)= & \iiint_{0}^{\infty} \delta\left(r-\alpha_{1} \alpha_{2}\right) \dot{r} f_{\dot{R} \mid r, \alpha}\left(\dot{r} \mid r, \alpha_{1}, \alpha_{2}\right) d \dot{r} \\
& \times f_{R \mid \alpha}\left(r \mid \alpha_{1}, \alpha_{2}\right) f_{12}\left(\alpha_{1}, \alpha_{2}\right) d \alpha_{1} d \alpha_{2} \\
= & \int_{0}^{\infty}\left(\int_{0}^{\infty} \dot{r} f_{\dot{R} \mid \alpha}\left(\dot{r} \mid \frac{r}{\alpha_{2}}, \alpha_{2}\right) d \dot{r}\right) \frac{1}{\alpha_{2}} f_{12}\left(\frac{r}{\alpha_{2}}, \alpha_{2}\right) d \alpha_{2},
\end{aligned}
$$


where $\delta(\cdot)$ is the Dirac delta function, $f_{12}\left(r / \alpha_{2}, \alpha_{2}\right)$ has the form given by (3) with $\alpha_{1}$ replaced by $r / \alpha_{2}$, and $f_{\dot{R} \mid \alpha}(\dot{r} \mid$ $\left.r / \alpha_{2}, \alpha_{2}\right)$ is given by (23) also with $\alpha_{1}$ replaced by $r / \alpha_{2}$. Substituting (18) into (22) and (3) into (24), we can express the normalized LCR in the single-integral form

$$
\begin{aligned}
\frac{N_{R}(r)}{f_{m}}= & \frac{\sqrt{\pi} r}{\sigma_{1}^{2} \sigma_{2}^{2}\left(1-\rho^{2}\right)} I_{0}\left(\frac{r|\rho|}{\sigma_{1} \sigma_{2}\left(1-\rho^{2}\right)}\right) \\
& \times \int_{0}^{\infty} \sqrt{\sigma_{1}^{2}-\frac{4 \sigma_{1}^{2} \sigma_{2}^{2}\left(\rho^{2}\right)^{\prime \prime}}{\alpha_{2}^{2}}+\frac{r^{2} \sigma_{2}^{2}}{\alpha_{2}^{4}}} \\
& \times e^{-\left(\left(\sigma_{1}^{2} \alpha_{2}^{2}+\sigma_{2}^{2} r^{2} / \alpha_{2}^{2}\right) / 2 \sigma_{1}^{2} \sigma_{2}^{2}\left(1-\rho^{2}\right)\right)} d \alpha_{2}
\end{aligned}
$$

that can be easily evaluated with a computing software, such as MATLAB. If $\rho=0$, it can be easily check that (25) will reduce to the form given in $[8,17,18]$ for independent cascaded Rayleigh channels.

3.2. AFD. Let $P(R \leq r)$ be the cumulative distribution function (cdf) of $R$. With the normalized LCR given by (25), the normalized AFD can be evaluated by [14]

$$
T_{R}(r)=f_{m} \cdot \frac{P(R \leq r)}{N_{R}(r)} .
$$

By using the pdf of $R$ given by (4), the cdf in the above AFD can be evaluated as

$$
\begin{aligned}
P(R \leq r)= & \int_{0}^{r} f_{R}(y) d y \\
= & \frac{1}{\sigma_{1}^{2} \sigma_{2}^{2}\left(1-\rho^{2}\right)} \int_{0}^{r} y I_{0}\left(\frac{y|\rho|}{\sigma_{1} \sigma_{2}\left(1-\rho^{2}\right)}\right) \\
& \times K_{0}\left(\frac{y}{\sigma_{1} \sigma_{2}\left(1-\rho^{2}\right)}\right) d y \\
= & 1-\frac{r|\rho|}{\sigma_{1} \sigma_{2}\left(1-\rho^{2}\right)} I_{1}\left(\frac{r|\rho|}{\sigma_{1} \sigma_{2}\left(1-\rho^{2}\right)}\right) \\
& \times K_{0}\left(\frac{r}{\sigma_{1} \sigma_{2}\left(1-\rho^{2}\right)}\right) \\
& -\frac{r}{\sigma_{1} \sigma_{2}\left(1-\rho^{2}\right)} I_{0}\left(\frac{r|\rho|}{\sigma_{1} \sigma_{2}\left(1-\rho^{2}\right)}\right) \\
& \times K_{1}\left(\frac{r}{\sigma_{1} \sigma_{2}\left(1-\rho^{2}\right)}\right),
\end{aligned}
$$

where $I_{1}(\cdot)$ is the first-order modified Bessel function of the first kind, and a formula modified from $[22,(6.521 .4)]$ with $I_{i}(z)=j^{-i} J_{i}(j z)(j=\sqrt{-1})$ is used to write the integral into a closed-form.

\section{Numerical Results of LCR and AFD}

From (17), due to the property of Bessel functions, $\rho^{2}$ is not a monotone function of $\tau$. However, as $\tau$ increases,

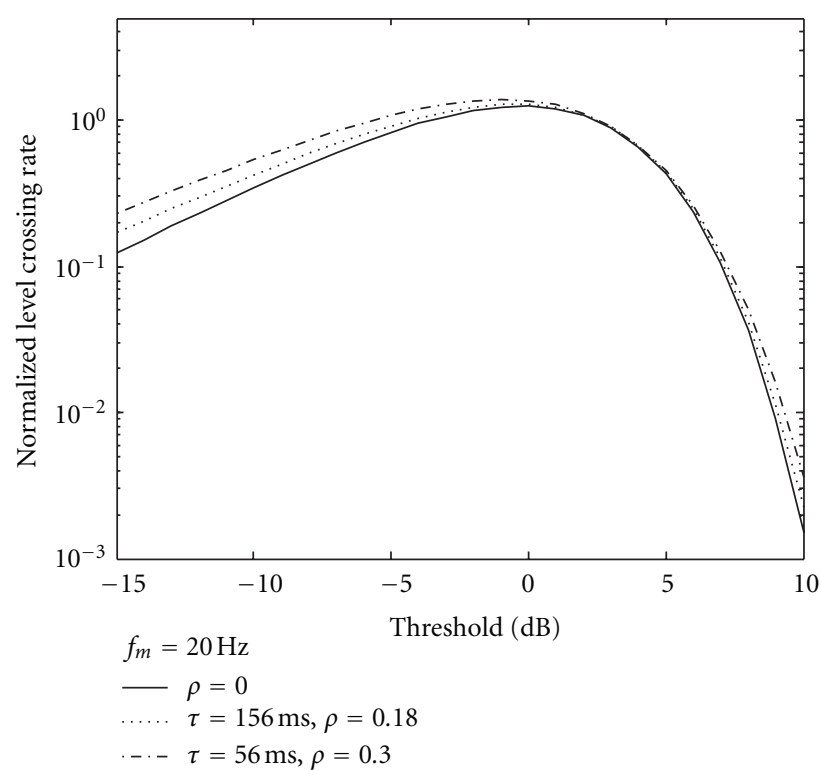

Figure 4: Normalized LCR for $\sigma_{1}^{2}=\sigma_{2}^{2}=1$.

the vibration range of $J_{0}^{2}\left(\omega_{m} \tau\right)$ tends to be smaller, and $\rho^{2}$ becomes smaller.

To demonstrate the impact of the correlation on the LCR and AFD, the normalized LCR of the correlated cascaded Rayleigh channels for different values of $\tau$ is plotted in Figure 4. The result in Figure 4 implies that when correlation increases, the cascaded envelope $R$ has more chances to enter a deep fading area. For example, as shown in Figure 4, the correlated cascaded channels may have a $5 \% \sim 10 \%$ more crossing rate than independent cascaded channels to go into deep fade below $-10 \mathrm{~dB}$.

In Figure 5, the numerical AFD is plotted. According to Figure 5, as the correlation increases, the average duration when $R$ temporarily stays in the deep fading area can be shorter although $R$ enters the area more frequently.

Thus, the correlation between cascaded channels not only affects the BER as shown in [10-13] but also changes the second-order statistics which are more serious in a deep fading area.

\section{Conclusions}

Different second-order correlation functions of completely correlated cascaded fading channels have been derived and the corresponding LCR and AFD are analyzed, which can facilitate the design of wireless communication or RFID systems on keyhole or pinhole channels. Under the consideration of the channel correlation, the LCR and AFD will depart from those obtained for independent cascaded channels. Numerical results show that the correlation has a higher impact on the second-order statistics in the deep fading region. The evaluation of LCR and AFD will also be helpful for the design of interleaving transmission and encoding schemes over correlated cascaded fading channels. 


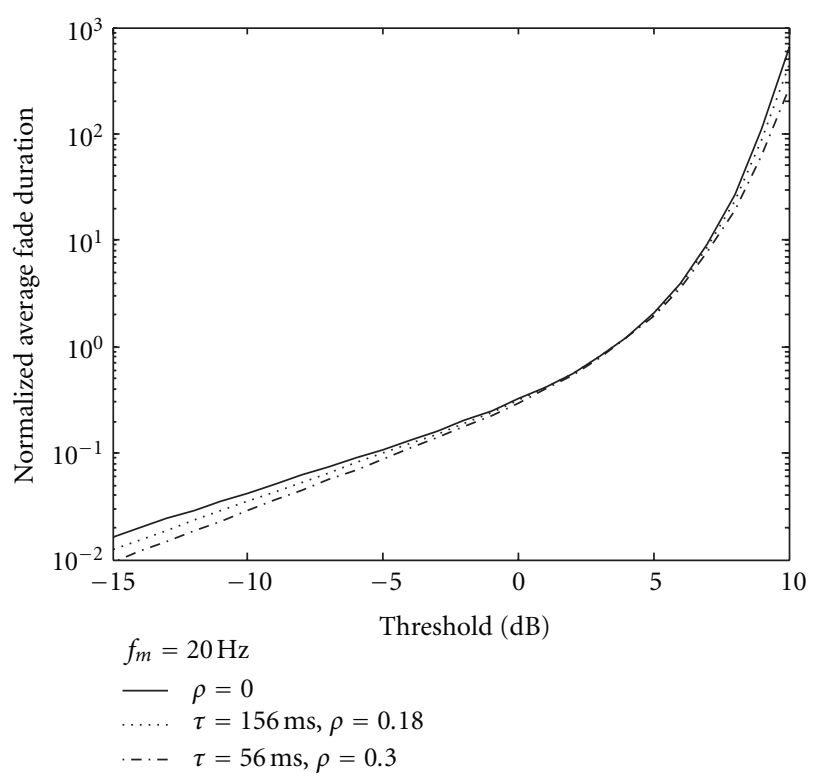

FIgURE 5: Normalized AFD for $\sigma_{1}^{2}=\sigma_{2}^{2}=1$.

Further research on related topics may consider the secondorder statistics of diversity schemes over correlated cascaded channels.

\section{Acknowledgment}

This paper was supported by the National Science Council, Taiwan, under Grant nos. NSC100-2220-E-155-001 and NSC100-2220-E-155-005.

\section{References}

[1] D. Gesbert, H. Bölcskei, D. A. Gore, and A. J. Paulraj, "Outdoor MIMO wireless channels: models and performance prediction," IEEE Transactions on Communications, vol. 50, no. 12, pp. 1926-1934, 2002.

[2] M. Uysal, "Diversity analysis of space-time coding in cascaded Rayleigh fading channels," IEEE Communications Letters, vol. 10, no. 3, pp. 165-167, 2006.

[3] G. K. Karagiannidis, N. C. Sagias, and P. T. Mathiopoulos, "N*Nakagami: a novel stochastic model for cascaded fading channels," IEEE Transactions on Communications, vol. 55, no. 8, pp. 1453-1458, 2007.

[4] D. Chizhik, G. J. Foschini, and R. A. Valenzuela, "Capacities of multi-element transmit and receive antennas: correlations and keyholes," Electronics Letters, vol. 36, no. 13, pp. 1099-1100, 2000.

[5] V. Erceg, S. J. Fortune, J. Ling, A. J. Rustako, and R. A. Valenzuela, "Comparisons of a computer-based propagation prediction tool with experimental data collected in urban microcellular environments," IEEE Journal on Selected Areas in Communications, vol. 15, no. 4, pp. 677-684, 1997.

[6] J. Salo, H. M. El-Sallabi, and P. Vainikainen, "The distribution of the product of independent Rayleigh random variables," IEEE Transactions on Antennas and Propagation, vol. 54, pp. 639-643, 2006.

[7] C. Zhu, J. Mietzner, and R. Schober, "On the performance of non-coherent transmission schemes with equal-gain combining in generalized K-fading," IEEE Transaction on Wireless Communications, vol. 9, no. 4, pp. 1337-1349, 2010.

[8] C. S. Patel, G. L. Stüber, and T. G. Pratt, "Statistical properties of amplify and forward relay fading channels," IEEE Transactions on Vehicular Technology, vol. 55, no. 1, pp. 1-9, 2006.

[9] C. He and Z. J. Wang, "Closed-form BER analysis of noncoherent FSK in MISO double Rayleigh fading/RFID channel," IEEE Communications Letters, vol. 15, no. 8, pp. 848-850, 2011.

[10] J. Griffin and G. Durgin, "Link envelope correlation in the backscatter channel," IEEE Communications Letters, vol. 11, no. 9, pp. 735-737, 2007.

[11] J. D. Griffin and G. D. Durgin, "Gains for RF tags using multiple antennas," IEEE Transactions on Antennas and Propagation, vol. 56, no. 2, pp. 563-570, 2008.

[12] C. He and Z. J. Wang, "Impact of the correlation between forward and backscatter channels on RFID system performance," in Proceedings of the IEEE International Conference on Acoustics, Speech and Signal Processing (ICASSP '11), pp. 35403543, May 2011.

[13] H. D. Goldman and R. C. Sommer, "An analysis of cascaded binary communication links," IRE Transactions on Communications Systems, vol. 10, no. 3, pp. 291-299, 1962.

[14] S. O. Rice, "Mathematical analysis of random noise," Bell System Technical Journal, vol. 23, pp. 282-332, 1944, pp. 46156, 1945.

[15] W. C. Jakes, Microwave Mobile Communications, IEEE Press, Piscataway, NJ, USA, 1994.

[16] Y. A. Chau and K. Y. Huang, "Burst-error analysis of dual-hop fading channels based on the second-order channel statistics," IEEE Transactions on Vehicular Technology, vol. 59, no. 6, pp. 3108-3115, 2010.

[17] Y. A. Chau and K. Y. Huang, "Channel statistics and performance of cooperative selection diversity with dual-hop amplify-and-forward relay over Rayleigh fading channels," IEEE Transactions on Wireless Communications, vol. 7, no. 5, pp. 1779-1785, 2008.

[18] N. Slatanov, Z. H. Velkov, and G. K. Karagiannidis, "Level crossing rate and average fade duration of the double Nakagami- $m$ random process and application in MIMO keyhole fading channels," IEEE Communications Letters, vol. 12, no. 11, pp. 822-824, 2008.

[19] Y. A. Chau and K. Y.-T. Huang, "Evaluation of level crossing rates and fade durations for multihop Nakagami fading channels with an amplify-and-forward relay," in Proceedings of the International Symposium on Intelligent Signal Processing and Communications Systems (ISPACS '07), pp. 732-735, Xiamen, China, November 2007.

[20] C. Floerkemeier and S. Sarma, "RFIDSim-a physical and logical layer simulation engine for passive RFID," IEEE Transactions on Automation Science and Engineering, vol. 6, no. 1, pp. 33-43, 2009.

[21] M. K. Simon, Probability Distributions Involving Gaussian Random Variables: A Handbook For Engineers and Scientists, Kluwer Academic, Norwell, Mass, USA, 2002.

[22] I. S. Gradshteyn and I. M. Ryzhik, Table of Integrals, Series, and Products, Academic Press, New York, NY, USA, 6th edition, 2000.

[23] M. Yacoub, Foundations of Mobile Radio Engineering, CRC Press, Boca Raton, Fla, USA, 1993.

[24] A. Papoulis, Probability, Random Variables, and Stochastic Processes, McGraw-Hill, New York, NY, USA, 3rd edition, 1991. 

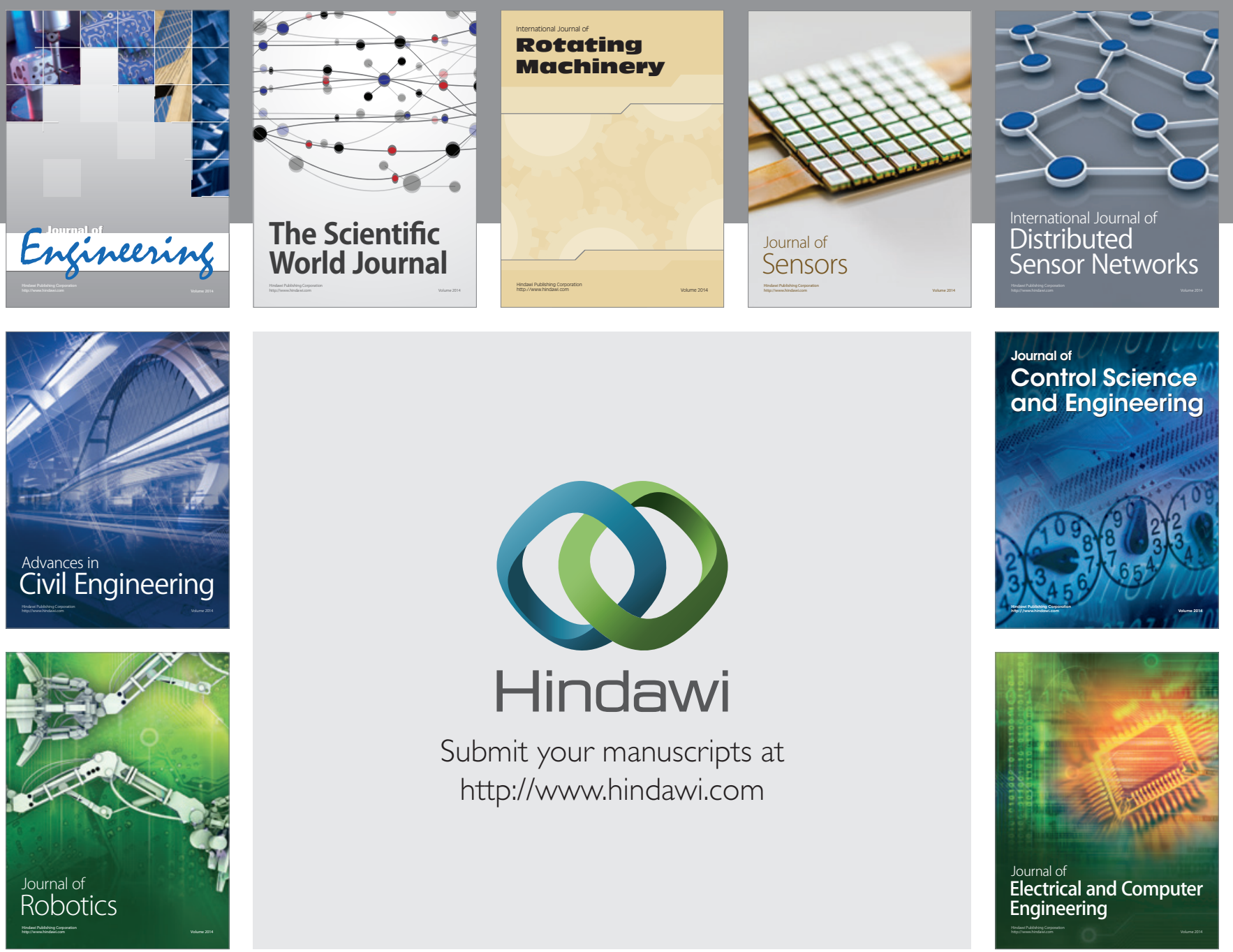

Submit your manuscripts at

http://www.hindawi.com
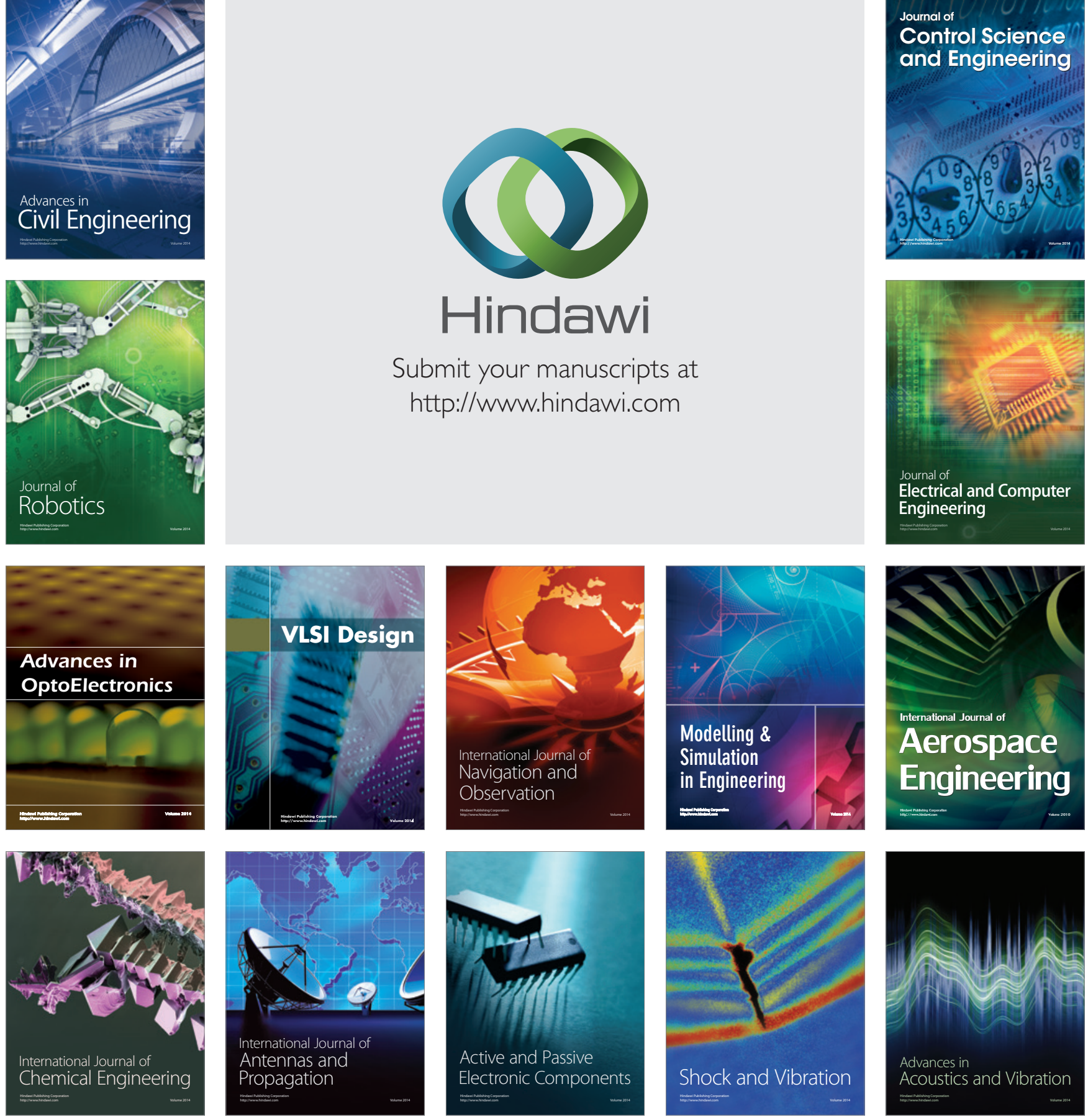